\title{
GCU
}

Glasgow Caledonian

University

University for the Common Good

\section{Establishing a baseline for a national paediatric antimicrobial stewardship programme}

Gibbons, C.L.; Malcolm, W.; Sneddon, J.; Doherty, C.; Cairns, S.; Milne, A.; Llano, M.; Reilly, J.S.

Published in:

Journal of Antimicrobial Chemotherapy

DOI:

10.1093/jac/dkz291

Publication date:

2019

Document Version

Author accepted manuscript

Link to publication in ResearchOnline

Citation for published version (Harvard):

Gibbons, CL, Malcolm, W, Sneddon, J, Doherty, C, Cairns, S, Milne, A, Llano, M \& Reilly, JS 2019, 'Establishing a baseline for a national paediatric antimicrobial stewardship programme', Journal of Antimicrobial

Chemotherapy, vol. 74, no. 10, pp. 3104-3110. https://doi.org/10.1093/jac/dkz291

\section{General rights}

Copyright and moral rights for the publications made accessible in the public portal are retained by the authors and/or other copyright owners and it is a condition of accessing publications that users recognise and abide by the legal requirements associated with these rights.

Take down policy

If you believe that this document breaches copyright please view our takedown policy at https://edshare.gcu.ac.uk/id/eprint/5179 for details of how to contact us. 
CL Gibbons ${ }^{1}$, W Malcolm ${ }^{* 1}$, J Sneddon ${ }^{2}$, C Doherty ${ }^{3}$, S Cairns ${ }^{1}$, A Milne ${ }^{1}$, M Llano ${ }^{1}$, JS Reilly ${ }^{1,4 t}$

4

\section{Affiliations}

6

7

8

9

10

11

12

${ }^{1}$ Health Protection Scotland, National Services Scotland, Meridian Court, 5 Cadogan Street, Glasgow, G2 6QE, UK.

${ }^{2}$ Healthcare Improvement Scotland, Delta House, 50 West Nile Street, Glasgow, G1 2NP, UK.

${ }^{3}$ Royal Hospital for Children, NHS Greater Glasgow and Clyde, 1345 Govan Rd, Glasgow G51 4TF, UK.

${ }^{4}$ Glasgow Caledonian University, School of Health and Life Sciences, Cowcaddens Road, Glasgow G4 OBA, UK

*Corresponding author

\section{A short running title}

Establishing a baseline for a national paediatric antimicrobial stewardship programme

\section{Word count: 2743}

\footnotetext{
${ }^{\dagger}$ Clinical Leadership \& Support, NHS National Services Scotland, Gyle Square, 1 South Gyle Crescent, Edinburgh, EH12 9EB. UK
} 


\section{Abstract}

Background: The majority of antimicrobial stewardship programmes (ASP) focus on prescribing in adult populations, however there is a recognised need for targeted paediatric antimicrobial stewardship to improve the quality and safety of prescribing amongst this patient group.

Objectives: To describe the current epidemiology of antimicrobial prescribing in paediatric inpatient populations in Scotland to establish a baseline of evidence and identify priority areas for quality improvement to support a national paediatric antimicrobial stewardship programme.

Methods: A total of 559 paediatric inpatients were surveyed during the Scottish national point prevalence survey of healthcare-associated infections and antimicrobial prescribing, 2016. The prevalence of antimicrobial prescribing was calculated and characteristics of antimicrobial prescribing were described as proportions and compared between specialist hospitals and paediatric wards in acute hospitals.

Results: Prevalence of antimicrobial use in paediatric inpatients was $35.4 \%$ (95\% Cl: 31.6 to 39.4). Treatment of community and hospital-acquired infections accounted for $47.1 \%$ and $20.7 \%$ of antimicrobial use respectively, with clinical sepsis being the most common diagnosis and gentamicin the most frequently prescribed antimicrobial for the treatment of infection. The reason for prescribing was documented in the notes for $86.5 \%$ of all prescriptions and of those assessed for compliance against local policy, 92.9\% were considered compliant.

Conclusions: Data from national prevalence surveys are advantageous when developing antimicrobial stewardship programmes (ASP). Results have highlighted differences in the prescribing landscape between paediatric inpatient populations in specialist hospitals and acute hospitals, and have informed priorities for the National ASP which reinforces the need for a targeted paediatric ASP. 
Introduction

Appropriate prescribing is imperative so that patients receive the optimal choice of antimicrobial to

treat or prevent infection, but also to avoid unfavourable consequences of antimicrobial misuse

including the emergence of multi-drug resistant pathogens, the risk of Clostridioides difficile

infection and other adverse events. ${ }^{1 ; 2}$ Antimicrobial stewardship programmes (ASP), which involve a coordinated approach to promoting and monitoring the careful use of antimicrobials to preserve their future effectiveness ${ }^{3}$, have been successful in improving antimicrobial prescribing behaviours and outcomes. ${ }^{1 ; 4}$

Until now, the majority of ASP have focused primarily on adults, however the need for a targeted ASP within paediatric populations is recognised. ${ }^{1}$ While there is evidence suggesting that prescribing within paediatric populations can often be inappropriate and with much variation between facilities; ${ }^{1 ; 5-7}$ it has been demonstrated that paediatric ASP can be successful in reducing overall antimicrobial use, costs and prescribing errors. No apparent negative impact on patient safety has been demonstrated, however more studies on outcomes is required ${ }^{7-9}$

This said, there are many complexities associated with paediatric antimicrobial stewardship including issues around early identification and diagnosis of a sick child. ${ }^{10}$ Signs and symptoms of infection vary greatly between newborns, infants, children and teenagers meaning that recognition and severity scoring are problematic which makes appropriate prescribing more challenging. ${ }^{10}$ Moreover, for quantitative surveillance of antimicrobial use within ASP, the internationally recognised standard unit of medicine consumption, the total DDD per 1000 population per day (DDD/1000/day), is not appropriate for use within paediatric populations. ${ }^{11}$ In the absence of continuous audit data, point prevalence surveys (PPS) have been used to successfully monitor the quality of antimicrobial prescribing in hospitals. ${ }^{12-14}$ The Antibiotic 
Resistance and Prescribing in European Children (ARPEC) study collected antimicrobial prescribing data from 17,693 paediatric patients in 226 hospitals in 41 countries worldwide using a standardised web-based prevalence methodology over one day in October and November $2012 .{ }^{15 ; 16}$ The ARPEC study identified a prescribing prevalence of $36.7 \%$ and described the feasibility of adapting existing adult quality indicators of antimicrobial prescribing for the paediatric hospital setting, with the purpose of uniformly assessing prescribing, identifying areas of poor practice and proposing international benchmarks. ${ }^{16}$ The Scottish Antimicrobial Prescribing Group (SAPG) was established in 2008 to coordinate the delivery of a national ASP to optimise antimicrobial prescribing. Using data from the Scottish national PPS, SAPG previously identified targets for national quality improvement in antimicrobial use in Scottish hospitals, ${ }^{13}$ however these stewardship efforts hitherto have focused on adult inpatients. The recent European-wide PPS of healthcare associated infection (HAI) and antimicrobial prescribing, 2016 provided a snapshot of the number and types of antimicrobials being prescribed, to whom and for which diagnosis at a single point in time. In Scotland, this survey was mandatory and conducted in all NHS acute and specialised hospitals, and therefore captured information on all hospitalised inpatients. ${ }^{17}$ Using these data, we aim to describe the current national prevalence of antimicrobial prescribing in paediatric inpatients in Scottish hospitals, to establish a baseline and identify priority areas for quality improvement as part of a national paediatric ASP, coordinated by SAPG.

Methods

91 Ethics

92 A Privacy Impact Assessment was undertaken and the project was reviewed and approved by the Public Benefit and Privacy Panel for Health and Social Care (PBPP) (Application Number: 1516-0599). 
PBPP is a governance structure of NHS Scotland that has authority for decision-making on behalf of NHSScotland Chief Executive Officers and the Registrar General.

Study design

97 Data from the Scottish national PPS of HAI and antimicrobial prescribing, 2016 were used. These data were collected during a rolling PPS conducted from $1^{\text {st }}$ September to $30^{\text {th }}$ November 2016 in all acute NHS hospitals in Scotland using a standardised methodology and definitions adapted for use in Scotland ${ }^{17}$ from the ECDC protocol. ${ }^{18}$ Data were collected in each hospital within a period of one week by trained staff from local infection prevention and control, and antimicrobial management teams. Data on inpatient demographics, $\mathrm{HAl}$ and antimicrobial prescribing were extracted from a number of sources available on the ward at the time of survey including prescribing charts, nursing and medical notes. Full details of the study design, inclusion/exclusion criteria and case definitions are described in the survey protocol. ${ }^{19}$

Data items collected and definitions

This analysis was restricted to paediatric inpatients only, which were defined as patients admitted to; (1) one of the three specialist paediatric NHS hospitals in Scotland or; (2) one of 22 acute NHS hospitals (teaching, general and maternity hospitals, herein described as 'acute') and were (a) aged 16 years and under or (b) aged 18 years and under, and under the care of a paediatric consultant. Healthy neonates were excluded from this analysis. Information on inpatient demographics, McCabe score (reflecting the underlying medical condition and based on prognosis) ${ }^{20}$ device use and specialty of care was collected for each surveyed patient. Data on systemic antibacterials (ATC J01) and antimycotics (J02) prescribed for treatment or prophylaxis were also collected. Antimycobacterials specifically for the treatment of Mycobacterium tuberculosis (J04), antivirals (J05) and topical antimicrobials were excluded. 
117 Antimicrobial prescribing prevalence was defined as the total number of paediatric inpatients receiving at least one systemic antimicrobial at the time of survey as a percentage of the total number of paediatric inpatients. The name of antimicrobial, route of administration, indication for prescribing and diagnosis were recorded for each antimicrobial. Diagnoses and indications were as recorded at the time of prescribing by the prescriber. Clindamycin, ciprofloxacin and other quinolones, co-amoxiclav and the cephalosporins were considered broad spectrum in this analysis, and the carbapenems and piperacillin/tazobactam considered very broad spectrum.

Information on any change of antimicrobial during a hospital stay; if the reason for prescribing was recorded in the notes or medicine chart and; if prescribing complied with local policy, was also collected. The percentage of antimicrobials with the reason recorded in the notes was calculated (excluding those where the patient's case notes were not available to review). The choice of antimicrobial prescribed for empirical prescribing and surgical prophylaxis was assessed to identify if this complied with local policy, if there was one available (the route, dose and duration were not assessed). Prescribing was considered non-compliant where the reason or indication was not recorded, and if notes were not available for review then policy was recorded as 'not known'. indicators of antimicrobial prescribing were also collected, including those collected as part of the ECDC protocol, and additional data to assess compliance with local policy

\section{Statistical analysis}

137 Inpatient demographics, McCabe score, device use and specialty of care were compared between 138 hospital types using Pearson's chi square tests with a continuity correction to provide an understanding of potential differences in prescribing patterns and the need for tailored ASP. The distribution of age between inpatients in specialist versus acute hospitals was compared using a Mann-Whitney U test. Prevalence calculations with Wilson's 95\% Cl were calculated. The prescribing 
quality indicators in specialist and acute hospitals were compared using Pearson's chi square tests. Analysis was carried out using $\mathrm{R}$ version 3.5.0.

Results

Survey population

147 A total of 559 paediatric inpatients were surveyed and the demographic details are described in Table 1. Approximately one half ( $n=249,44.5 \%$ ) of paediatric inpatients received care in one of the three specialist paediatric hospitals, and the remainder received care within one of 22 acute hospitals. Patients in specialist hospitals were older $(p<0.001)$, had poorer prognoses $(p<0.001)$, had higher use of central vascular catheters $(p<0.001)$ and urinary catheter use $(p=0.02)$ when compared with those in acute hospitals.

\section{Prevalence of antimicrobial use}

A total of 200 paediatric inpatients received 368 antimicrobials, giving an antimicrobial prescribing prevalence of $35.9 \%$ (95\% Cl: 32.0 to 40.0 ). The prevalence of prescribing in paediatric specialist hospitals (40.7\%, 95\% Cl: 34.8 to 46.9$)$ was significantly higher than in acute hospitals $(32.0 \%, 95 \%$ Cl: 27.1 to 37.4$)(p=0.03)$.

\section{Characteristics of antimicrobial prescribing}

161 Two thirds of antimicrobials prescribed were for treatment of infection. Community acquired 162 infection (47.8\%) and hospital acquired infection (20.6\%) were the most frequent indications for 163 prescribing Medical prophylaxis and surgical prophylaxis accounted for $20.9 \%$ and $1.2 \%$ of all antimicrobials prescribed, respectively. 
Five diagnoses accounted for over half of all antimicrobials prescribed for treatment of infection: clinical sepsis, febrile neutropaenia, intra-abdominal sepsis, bronchitis and cystic fibrosis (Table 2). The types of infections treated differed in acute and specialist hospitals with clinical sepsis and febrile neutropaenia the most common diagnoses, respectively. The overall prevalence of antimicrobial prescribing for the treatment of clinical sepsis among all paediatric inpatients was $4.8 \%$ (95\% Cl: 3.4 to 7.0 ). There were 27 paediatric inpatients receiving 36 antimicrobials for general medical prophylaxis of unspecified anatomical site, e.g. medical prophylaxis in haematology patients, which was the most frequently recorded reason. Four inpatients received a total of four antimicrobials for surgical prophylaxis.

The distribution of antimicrobial agents, by indication, is shown in Figure 1. Gentamicin was the most frequently prescribed antimicrobial for the treatment of infection (15.8\%), and ten different antimicrobial types accounted for more than three quarters of all treatment antimicrobials prescribed. Co-trimoxazole was the most commonly prescribed antimicrobial for medical prophylaxis (23.2\%). Very broad spectrum antimicrobials accounted for $9.0 \%$ of all antimicrobials prescribed. This was higher in specialist paediatric hospitals than acute hospitals ( $12.4 \%$ versus $5.5 \%, p=0.02)$. Broad spectrum antimicrobials, commonly associated with Clostridioides difficile infection, accounted for one in five antimicrobials prescribed and there was no significant difference between specialist and acute hospitals.

Three quarters of antimicrobials were administered via the parenteral route, with clinical sepsis and intra-abdominal sepsis being the most common diagnoses treated parenterally. More than a third (35.5\%) of parenteral antimicrobials had been given for longer than three days at the time of the survey. Two fifths of oral antimicrobials had been given for more than seven days at the the time of survey $(n=36,40.0 \%)$.

Of the 368 antimicrobials prescribed to paediatric inpatients, the antimicrobial agent or route of administration was changed and the reason for change documented for 54 antimicrobials. The 
reasons for change included: escalation of therapy for $55.6 \%$ of antimicrobials $(n=30)$, intravenous to oral switch for $20.4 \%$ of antimicrobials ( $n=11$ ), and de-escalation for $11.1 \%$ of antimicrobials $(n=6)$.

192 The reason for change of the remainder was described as 'other'.

193 The reason for prescribing was documented in the notes for $85.8 \%(n=307)$ of all prescriptions and for $68.9 \%$ and $91.8 \%$ of prophylactic and treatment antimicrobials, respectively. For antimicrobials assessed for compliance with local policy, $92.1 \%$ were considered compliant. This was $100.0 \%$ and $92.0 \%$ for surgical prophylaxis $(n=2)$ and treatment $(n=163)$ respectively, and $86.8 \%$ and $95.9 \%$ in paediatric specialist and acute hospitals, respectively.

Antimicrobial prescribing quality indicators adapted for Scotland from those suggested by ARPEC, are described in Table 3. The percentage of antimicrobials where the reason for prescribing was documented and compliance with local policy were higher in the acute hospitals compared with the specialist hospitals ( $p<0.001$ and $p=0.03$, respectively). 


\section{Discussion}

This study is, as far as we know, the first comprehensive national point prevalence survey of antimicrobial prescribing and antimicrobial indicators in all hospitalised paediatric patients at the national level. These data provide the baseline to inform the establishment of a national paediatric antimicrobial stewardship programme.

Our findings estimate that around one in every three hospitalised paediatric patients in Scotland receive antimicrobial treatment. While the prevalence of antimicrobial prescribing is similar to that in adults in acute hospitals in Scotland ${ }^{17}$ the prescribing landscape differs. For example, approximately one fifth of treatment antimicrobials prescribed in paediatric populations was for a diagnosis of clinical sepsis whereas by contrast, this accounted for only $2.9 \%$ of the treatment antimicrobials prescribed to adults in acute hospitals. Similarly, approximately one in every five antimicrobials prescribed to paediatric patients were for medical prophylaxis, but in the adult population this figure was one in twenty. These are important differences that justify the need for a targeted paediatric ASP. The high prevalence of clinical sepsis highlights the importance of severity scoring and use of prognostic indicators in paediatric inpatients. This is important in the context of fewer community-acquired sepsis cases due to improved immunisation schedules. Since the recognition of clinical sepsis in children can often be challenging and their response to treatment variable, regular clinical reviews should feature as a key part of stewardship activities.

We found differences between acute hospitals and specialist hospitals which may reflect that alongside other local improvement initiatives, the Scottish national antimicrobial stewardship programme may be having a broader influence than where it has been targeted in acute adult inpatient populations. We found that the reason for prescribing was documented for $86.5 \%$ of all prescribed antimicrobials although this varied by indication $(72.6 \%$ and $91.8 \%$ for prophylaxis and 
treatment antimicrobials, respectively) and hospital type (79.9\% and $92.3 \%$ for specialist and acute hospitals, respectively). Documenting the reason for prescribing in patient notes is important to ensure that all health professionals involved in giving care to a patient are aware of the current and historical clinical and prescribing picture. ${ }^{16}$ While this level of documentation is encouraging considering that national interventions have focused on adult populations, these data highlight a potential area for improvement, particularly around medical prophylaxis.

Similarly we found compliance with local policy in paediatric patients in acute hospitals was higher than in specialist hospitals (95.9\% versus $86.8 \%$ ). Compliance with local prescribing policies is a key feature of ASP and ensures that prescribing is evidence-based and takes account of local antimicrobial resistance patterns. This suggests that a more individualised approach to antimicrobial therapy is used in specialist hospitals, which may be driven by differences in staffing and expertise. Further work is required to better understand the reasons for non-compliance in these hospitals. There were a number of differences with respect to the paediatric population and antimicrobial prescribing patterns between specialist hospitals and paediatric wards within acute hospitals. Patients in specialist hospitals require more specialised care and therefore different stewardship activities may need to be considered depending on the hospital setting.

Three quarters of antimicrobials were administered via the parenteral route and more than a third of these were administered for longer than three days at the time of survey. Parenterally administered antimicrobials are commonly employed in paediatrics as unwell and particularly younger children are often unable to tolerate oral antimicrobials. However, a key stewardship intervention is the regular review of patients prescribed parenteral antimicrobials to consider stopping treatment or an intravenous (IV) to oral switch when clinically appropriate. ${ }^{21}$ 
One in every six antimicrobials prescribed to paediatric patients was considered to be broad spectrum and one in every 12 was very broad spectrum. The use of broad spectrum antimicrobials is associated with an increased risk of Clostridioides difficile infection (CDI) and reducing inappropriate use of these agents continues to be a priority. In addition, many of the antimicrobial agents considered broad and very broad spectrum including carbapenems, piperacillin/tazobactam, third generation cephalosporins, macrolides and quinolones are on the World Health Organisation's 'watch' list due to concerns over higher toxicity and antimicrobial resistance potential. ${ }^{2}$ Their use should be restricted to preserve their activity and only prescribed on specialist advice with the ongoing need for them be reviewed on a daily basis.

\section{Strengths and limitations}

A key strength of this analysis is that the PPS included all NHS hospitals in Scotland thereby giving a complete national picture of prescribing in the paediatric inpatient population. Additionally, the protocol and case definitions used were evidence-based and internationally agreed giving comparability and methodological rigour. Limitations of all prevalence surveys are that they represent a snapshot of prescribing at one point in time which may not represent prescribing at other times, trends cannot be measured and causality cannot be inferred. Furthermore, the survey included 559 paediatric inpatients however, due to missing information for some data items, not all inpatients could be included in denominators when calculating prevalence estimates or percentages.

Conclusion

The availability of current data and quality indicators from a national prevalence survey that includes the whole population is an advantage when priority setting and developing antimicrobial stewardship programmes. In this study we investigated patterns of antimicrobial use and described potential quality indicators that provide baseline information on antimicrobial use in paediatric populations. We demonstrated differences in AM use between acute and specialist hospitals and, 
SAPG as part of the national paediatric ASP is addressing the variation through development of national consensus guidelines. We will utilise experience in specialist hospitals to optimise management of common infections in children across all settings. Additional key clinical priority areas include working with patient safety partners to improve recognition of sepsis in young children and consideration for the implementation of quality imrovement toolkit to support review of parenteral therapy, duration of oral treatment.

\section{Acknowledgements}

We would like thank the local Infection Prevention and Control Teams and Antimicrobial

Management Teams in the participating hospitals for conducting data collection and supporting this project.

\section{Funding}

This work was funded by the Scottish Government.

\section{Transparency declarations}

None to declare.

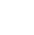

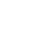


(1) Principi N, Esposito S. Antimicrobial stewardship in paediatrics. [Review]. BMC Infectious Diseases 2016 Aug 18;16(1):424.

(2) Sharland M, Pulcini C, Harbarth S, Zeng M, Gandra S, Mathur S, et al. Classifying antibiotics in the WHO Essential Medicines List for optimal use\&\#x2014; be AWaRe. The Lancet Infectious Diseases 2018 Jan 1;18(1):18-20.

(3) National Institute for Health and Care Excellence. Antimicrobial stewardship: systems and processes for effective antimicrobial medicine use. NICE 2015 AugustAvailable from: URL: https://www.nice.org.uk/guidance/ng15

(4) Dortch MJ, Fleming SB, Kauffmann RM, Dossett LA, Talbot TR, May AK. Infection reduction strategies including antibiotic stewardship protocols in surgical and trauma intensive care units are associated with reduced resistant gram-negative healthcare-associated infections. Surgical Infections 2011 Feb;12(1):15-25.

(5) Levy ER, Swami S, Dubois SG, Wendt R, Banerjee R. Rates and appropriateness of antimicrobial prescribing at an academic children's hospital, 2007-2010. Infection Control \& Hospital Epidemiology 2012 Apr;33(4):346-53.

(6) Nichols K, Stoffella S, Meyers R, Girotto J, on behalf of the Advocacy Committee for the Pediatric Pharmacy Advocacy Group. Pediatric Antimicrobial Stewardship Programs. J Pediatr Pharmacol Ther 2017 Jan;22(1):77-80.

(7) Smith MJ, Gerber JS, Hersh AL. Inpatient Antimicrobial Stewardship in Pediatrics: A Systematic Review. [Review]. Journal of the Pediatric Infectious Diseases Societ 2015 Dec;4(4):e127-e135.

(8) Hersh AL, De Lurgio SA, Thurm C, Lee BR, Weissman SJ, Courter JD, et al. Antimicrobial stewardship programs in freestanding children's hospitals. Pediatrics 2015 Jan;135(1):33-9.

(9) Oberje EJM, Tanke MAC, Jeurissen PPT. Antimicrobial Stewardship Initiatives Throughout Europe: Proven Value for Money. Infectious Disease Reports 2017 Mar 30;9(1):6800.

(10) Gerber JS, Kronman MP, Ross RK, Hersh AL, Newland JG, Metjian TA, et al. Identifying Targets for Antimicrobial Stewardship in Children's Hospitals. 2013;2015/01/01(12):1252-8.

(11) Porta A, Hsia Y, Doerholt K, Spyridis N, Bielicki J, Menson E, et al. Comparing neonatal and paediatric antibiotic prescribing between hospitals: a new algorithm to help international benchmarking. Journal of Antimicrobial Chemotherapy 2012 May;67(5):1278-86.

(12) Dean B, Lawson W, Jacklin A, Rogers T, Azadian B, Holmes A. The use of serial pointprevalence studies to investigate hospital anti-infective prescribing. International Journal of Pharmacy Practice 2002;10(2):121-5.

(13) Malcolm W, Nathwani D, Davey P, Cromwell T, Patton A, Reilly J, et al. From intermittent antibiotic point prevalence surveys to quality improvement: experience in Scottish hospitals. Antimicrobial Resistance \& Infection Control 2013 Jan 15;2(1):3. 
(14) Willemsen I, Groenhuijzen A, Bogaers D, Stuurman A, van KP, Kluytmans J. Appropriateness of antimicrobial therapy measured by repeated prevalence surveys. Antimicrobial Agents \& Chemotherapy 2007 Mar;51(3):864-7.

(15) Hufnagel M, Versporten A, Bielicki J, Drapier N, Sharland M, Goossens H, et al. High Rates of Prescribing Antimicrobials for Prophylaxis in Children and Neonates: Results From the Antibiotic Resistance and Prescribing in European Children Point Prevalence Survey. Journal of the Pediatric Infectious Diseases Society 2018 Mar 22;iy019.

(16) Versporten A, on behalf of the ARPEC project group, Bielicki J, on behalf of the ARPEC project group, Drapier N, on behalf of the ARPEC project group, et al. The Worldwide Antibiotic Resistance and Prescribing in European Children (ARPEC) point prevalence survey: developing hospital-quality indicators of antibiotic prescribing for children. Journal of Antimicrobial Chemotherapy 2016 Apr 1;71(4):1106-17.

(17) Health Protection Scotland. National Point Prevalence of Healthcare Associated Infection and Antimicrobial Prescribing 2016. HPS 2017 May 23

(18) European Centre for Disease Prevention and Control. Point prevalence survey of healthcare associated infections and antimicrobial use in European acute care hospitals. Protocol version 5.2. ECDC 2016 MayAvailable from: URL: https://ecdc.europa.eu/en/publicationsdata/point-prevalence-survey-healthcare-associated-infections-and-antimicrobial-use-3

(19) Health Protection Scotland. Point Prevalence Survey of Healthcare Associated Infection and Antimicrobial Prescribing 2016: Protocol for the collection of patient and ward level data. HPS 2017 MayAvailable from: URL: https://www.hps.scot.nhs.uk/resourcedocument.aspx?id=5968

(20) McCABE WR, JACKSON G. Gram-negative bacteremia: I. etiology and ecology. Archives of Internal Medicine 1962 Dec 1;110(6):847-55.

(21) Barlam TF, Cosgrove SE, Abbo LM, MacDougall C, Schuetz AN, Septimus EJ, et al. Implementing an Antibiotic Stewardship Program: Guidelines by the Infectious Diseases Society of America and the Society for Healthcare Epidemiology of America. Clinical Infectious Diseases 2016 May 15;62(10):e51-e77. 
Figure 1: Distribution of antimicrobials agents prescribed to the hospitalised paediatric population, by indication, 2016

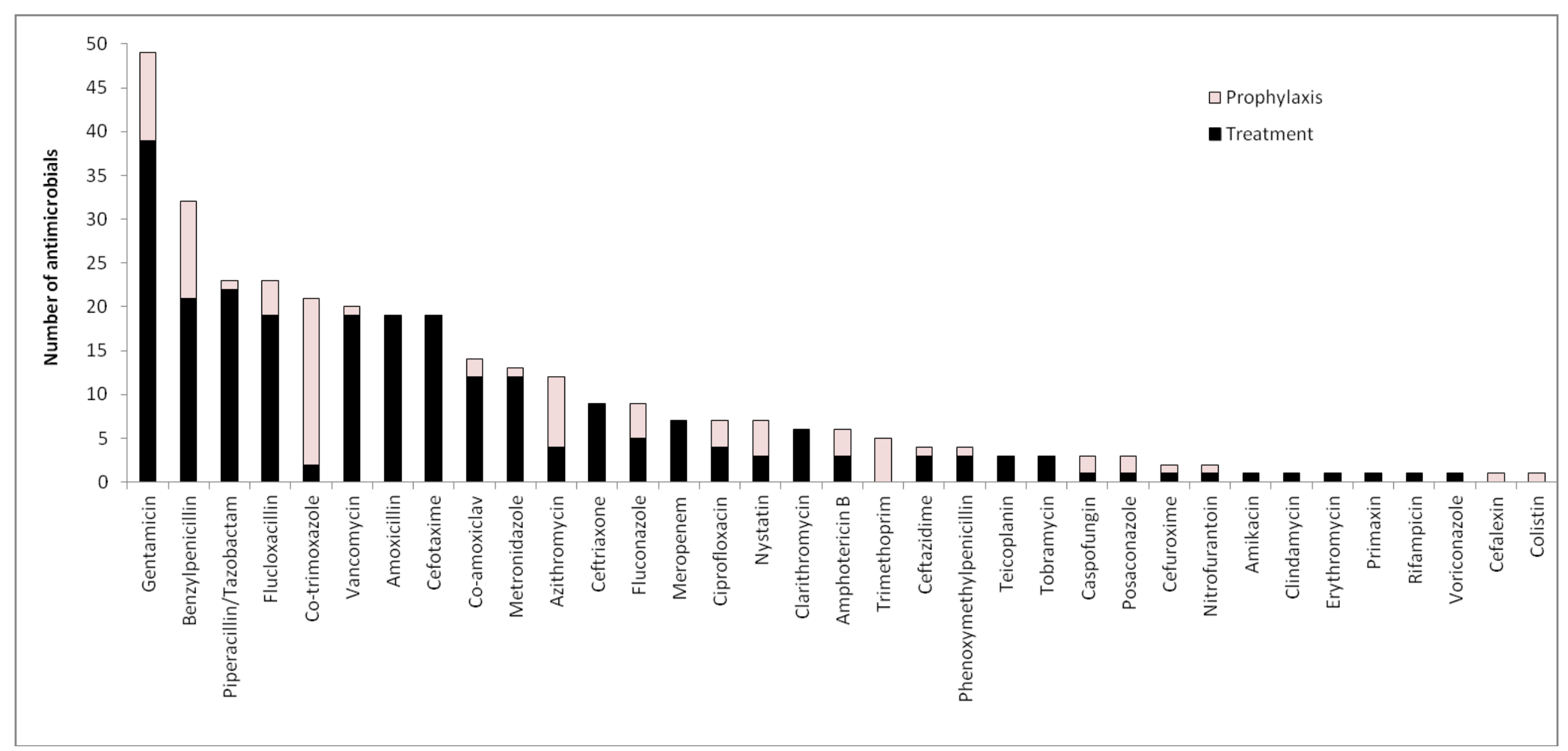


Table 1: Characteristics of the paediatric inpatient population, 2016

\begin{tabular}{|c|c|c|c|c|}
\hline & & $\begin{array}{c}\text { Paediatric inpatients in } \\
\text { acute hospitals }\end{array}$ & $\begin{array}{c}\text { Paediatric inpatients in } \\
\text { specialist hospitals }\end{array}$ & All paediatric inpatients \\
\hline & Number of patients & $\mathrm{n}=310$ & $n=249$ & $\mathrm{n}=559$ \\
\hline \multirow[t]{3}{*}{ Age } & Median age & $\begin{array}{c}1 \text { month (range } 0 \text { months } \\
\text { to } 17 \text { years, IQR = } 0 \\
\text { months to } 21 \text { months) }\end{array}$ & $\begin{array}{c}24 \text { months (range } 0 \text { months } \\
\text { to } 18 \text { years, IQR }=0 \text { months } \\
\text { to } 9 \text { years) }\end{array}$ & $\begin{array}{l}5 \text { months (range } 0 \text { months to } 18 \\
\text { years, IQR = } 0 \text { months to } 5 \text { years) }\end{array}$ \\
\hline & $\%$ aged $<1$ year & $71.6 \%$ & $34.1 \%$ & $54.9 \%$ \\
\hline & $\%$ aged $<1$ month & $42.6 \%$ & $12.0 \%$ & $29.0 \%$ \\
\hline Sex & $\%$ female & $45.8 \%$ & $44.2 \%$ & $45.1 \%$ \\
\hline Prognosis & \% with most severe McCabe Scores & $8.6 \%$ & $20.5 \%$ & $14.1 \%$ \\
\hline \multirow{4}{*}{ Devices } & \% with CVC in situ & $6.1 \%$ & $27.3 \%$ & $15.6 \%$ \\
\hline & \% with PVC in situ & $33.8 \%$ & $40.1 \%$ & $36.6 \%$ \\
\hline & $\%$ with urinary catheter in situ & $1.0 \%$ & $4.5 \%$ & $2.5 \%$ \\
\hline & \% with intubation & $5.8 \%$ & $9.3 \%$ & $7.4 \%$ \\
\hline \multirow{3}{*}{ Specialties } & \multirow{3}{*}{ Three most common specialties } & $\begin{array}{l}\text { Paediatric Neonatology } \\
\text { (other than NICU) }(35.8 \%)\end{array}$ & General Medicine (14.9\%) & $\begin{array}{c}\text { Paediatric Neonatology (other } \\
\text { than NICU) }(23.1 \%)\end{array}$ \\
\hline & & ICU Neonatal (27.1\%) & $\begin{array}{l}\text { ICU Neonatal (10.8\%) and } \\
\text { ICU Paediatric (10.8\%) }\end{array}$ & ICU Neonatal (19.9\%) \\
\hline & & General Medicine (14.2\%) & $\begin{array}{c}\text { General Surgery (Excluding } \\
\text { vascular) }(8.4 \%)\end{array}$ & General Medicine (14.5\%) \\
\hline
\end{tabular}


Table 2: Distribution of antimicrobials prescribed for treatment of infection in paediatric inpatients in 2016, by diagnosis

\begin{tabular}{|c|c|c|c|c|c|c|}
\hline \multirow{3}{*}{ Diagnosis } & \multicolumn{6}{|c|}{ Antimicrobials } \\
\hline & \multicolumn{2}{|c|}{$\begin{array}{c}\text { Paediatric inpatients in } \\
\text { acute hospitals }\end{array}$} & \multicolumn{2}{|c|}{$\begin{array}{c}\text { Paediatric inpatients in } \\
\text { specialist hospitals }\end{array}$} & \multicolumn{2}{|c|}{ All paediatric inpatients } \\
\hline & $\mathrm{N}$ & $\%$ & $\mathrm{~N}$ & $\%$ & $\mathrm{~N}$ & $\%$ \\
\hline Clinical sepsis (suspected BSI without lab confirmation), excluding febrile neutropaenia & 39 & 31.5 & 11 & 9.7 & 50 & 21.1 \\
\hline Febrile neutropaenia & 0 & 0.0 & 22 & 19.5 & 22 & 9.3 \\
\hline Intra-abdominal sepsis (including hepatobiliary) & 12 & 9.7 & 9 & 8.0 & 21 & 8.9 \\
\hline Acute bronchitis or exacerbations of chronic bronchitis & 7 & 5.7 & 10 & 8.9 & 17 & 7.2 \\
\hline Cystic fibrosis & 5 & 4.0 & 12 & 10.6 & 17 & 7.2 \\
\hline Pneumonia & 8 & 6.5 & 7 & 6.2 & 15 & 6.3 \\
\hline Cellulitis, wound, deep soft tissue not involving bone & 13 & 10.5 & 2 & 1.8 & 15 & 6.3 \\
\hline Infections of ear, nose, throat, larynx and mouth & 9 & 7.3 & 6 & 5.3 & 15 & 6.3 \\
\hline Symptomatic upper urinary tract infection & 7 & 5.7 & 6 & 5.3 & 13 & 5.5 \\
\hline Infections of the central nervous system & 7 & 5.7 & 6 & 5.3 & 13 & 5.5 \\
\hline Laboratory confirmed bacteraemia & 3 & 2.4 & 9 & 8.0 & 12 & 5.1 \\
\hline Systemic inflammatory response with no clear anatomic site & 7 & 5.7 & 4 & 3.5 & 11 & 4.6 \\
\hline Symptomatic lower urinary tract infection & 2 & 1.6 & 3 & 2.7 & 5 & 2.1 \\
\hline Surgical site infection involving skin or soft tissue but not bone & 1 & 0.8 & 3 & 2.7 & 4 & 1.7 \\
\hline Septic arthritis (including prosthetic joint), osteomyelitis & 2 & 1.6 & 0 & 0.0 & 2 & 0.8 \\
\hline Gastrointestinal infections & 0 & 0.0 & 2 & 1.8 & 2 & 0.8 \\
\hline Gynaecological infections & 1 & 0.8 & 0 & 0.0 & 1 & 0.4 \\
\hline Not recorded & 1 & 0.8 & 1 & 0.9 & 2 & 0.8 \\
\hline Total & 124 & 100.0 & 113 & 100.0 & 237 & 100.0 \\
\hline
\end{tabular}


Table 3: Comparison of quality indicators of antimicrobial prescribing in hospitalised paediatric populations between acute and specialist, 2016

\begin{tabular}{|c|c|c|c|c|}
\hline Quality indicators & $\begin{array}{c}\text { Prescribing in paediatric wards in } \\
\text { acute hospitals* } \\
\text { n=99 patients; } \\
n=183 \text { antimicrobials }\end{array}$ & $\begin{array}{l}\text { Prescribing in specialist } \\
\text { paediatric hospitals* } \\
\text { n=101 patients; } \\
n=185 \text { antimicrobials }\end{array}$ & $\begin{array}{l}\text { Comparison of prescribing } \\
\text { indicators in paediatric wards in } \\
\text { acute hospitals with prescribing } \\
\text { in specialist paediatric hospitals }\end{array}$ & $\begin{array}{l}\text { All paediatric prescribing* } \\
\begin{array}{l}n=200 \text { patients; } \\
n=368 \text { antimicrobials }\end{array}\end{array}$ \\
\hline $\begin{array}{l}\text { Documentation of the reason for antimicrobial } \\
\text { prescribing in the notes }\end{array}$ & $\begin{array}{c}91.6 \% \\
(n=164)\end{array}$ & $\begin{array}{c}79.9 \% \\
(n=143)\end{array}$ & $p<0.001$ & $\begin{array}{c}85.8 \% \\
(n=307)\end{array}$ \\
\hline $\begin{array}{l}\text { Percentage of all antimicrobials prescribed that } \\
\text { were compliant with local policy (empirical } \\
\text { prescribing and surgical prophylaxis) }\end{array}$ & $\begin{array}{l}95.9 \% \\
(n=93)\end{array}$ & $\begin{array}{l}86.8 \% \\
(n=59)\end{array}$ & $p=0.03$ & $\begin{array}{l}92.1 \% \\
(n=152)\end{array}$ \\
\hline $\begin{array}{l}\text { Percentage of all antimicrobials prescribed that } \\
\text { were administered via the parenteral route }\end{array}$ & $\begin{array}{c}82.8 \% \\
(n=149)\end{array}$ & $\begin{array}{c}64.1 \% \\
(n=116)\end{array}$ & $p<0.001$ & $\begin{array}{l}73.4 \% \\
(n=265)\end{array}$ \\
\hline $\begin{array}{l}\text { Percentage of patients receiving antimicrobials that } \\
\text { were receiving two or more (combination therapies) }\end{array}$ & $\begin{array}{l}66.7 \% \\
(n=66)\end{array}$ & $\begin{array}{l}50.5 \% \\
(n=51)\end{array}$ & $p=0.02$ & $\begin{array}{l}58.5 \% \\
(n=117)\end{array}$ \\
\hline $\begin{array}{l}\text { Percentage of all antimicrobials prescribed that } \\
\text { were broad spectrum or very broad spectrum }\end{array}$ & $\begin{array}{l}\text { BS: } 15.3 \%(n=28) \\
\text { VBS: } 5.5 \%(n=10)\end{array}$ & $\begin{array}{l}\text { BS: } 20.5 \%(n=38) \\
\text { VBS: } 12.4 \%(n=23)\end{array}$ & $\begin{array}{l}p=0.19 \\
p=0.02\end{array}$ & $\begin{array}{l}\text { BS: } 17.9 \%(n=66) \\
\text { VBS: } 9.0 \%(n=33)\end{array}$ \\
\hline $\begin{array}{l}\text { Antimicrobial prescribing prevalence for hospital- } \\
\text { acquired infections (excluding healthy neonates) }\end{array}$ & $\begin{array}{c}8.1 \% \\
(95 \% \mathrm{Cl}: 5.5 \text { to } 11.7) \\
(n=25)\end{array}$ & $\begin{array}{c}6.0 \% \\
(95 \% \text { Cl: } 3.7 \text { to } 9.7) \\
(n=15)\end{array}$ & $p=0.35$ & $\begin{array}{c}7.2 \% \\
(95 \% \text { Cl: } 5.3 \text { to } 9.6) \\
(n=40)\end{array}$ \\
\hline $\begin{array}{l}\text { Percentage of antimicrobials prescribed to treat } \\
\text { hospital-acquired infections* that were broad } \\
\text { spectrum or very broad spectrum }\end{array}$ & $\begin{array}{l}\text { BS: } 4.9 \%(n=2) \\
\text { VBS: } 17.1 \%(n=7)\end{array}$ & $\begin{array}{l}\text { BS: } 3.3 \%(n=1) \\
\text { VBS: } 20.0 \%(n=6)\end{array}$ & $\begin{array}{l}p=0.75 \\
p=0.75\end{array}$ & $\begin{array}{l}\text { BS: } 4.2 \%(n=3) \\
\text { VBS: } 18.3 \%(n=13)\end{array}$ \\
\hline $\begin{array}{l}\text { Percentage of antimicrobials prescribed to treat } \\
\text { community-acquired infections* that were broad } \\
\text { spectrum or very broad spectrum }\end{array}$ & $\begin{array}{l}\text { BS: } 26.8 \%(n=22) \\
\text { VBS: } 1.2 \%(n=1)\end{array}$ & $\begin{array}{l}\text { BS: } 27.7 \%(n=23) \\
\text { VBS: } 19.3 \%(n=16)\end{array}$ & $\begin{array}{l}p=0.90 \\
p<0.001\end{array}$ & $\begin{array}{l}\text { BS: } 27.3 \%(n=45) \\
\text { VBS: } 10.3 \%(n=17)\end{array}$ \\
\hline
\end{tabular}

Notes: *All percentages exclude records with missing information from denominator. BS: Broad spectrum antimicrobials - clindamycin, ciprofloxacin and other quinolones,

co-amoxiclav and the cephalosporins; VBS: Very broad spectrum - carbapenems and piperacillin/tazobactam 